\title{
Sensitivity to Bacillus subtilis: A Novel System for Selection of Heterozygous Diploids of Dictyostelium discoideum
}

\author{
By P. C. NEWELL, R. F. HENDERSON, D. MOSSES \\ AND D. I. RATNER \\ Department of Biochemistry, University of Oxford, \\ South Parks Road, Oxford $O X$ I $3 Q U$
}

(Received 8 November 1976; revised 20 December 1976)

\section{INTRODUCTION}

Genetic analysis of the cellular slime mould Dictyostelium discoideum makes use of the parasexual cycle. In this cycle haploid amoebae of two strains occasionally fuse to form heterozygous diploids that slowly and spontaneously revert to the haploid state with random segregation of the two sets of chromosomes (Sinha \& Ashworth, 1969; Brody \& Williams, 1974). This cycle allows complementation and linkage studies to be made (Pontecorvo \& Käfer, 1958; Katz \& Sussman, 1972). Spontaneous fusion to form heterozygous diploids, however, is a rare event, diploids being recovered at a frequency of about $\mathrm{I} \times \mathrm{IO}^{-5}$ in a population of starving amoebae over a $17 \mathrm{~h}$ period (Williams, Kessin \& Newell, I974a, $b$ ).

To overcome this problem, Loomis (1969) devised a selective method involving haploid strains carrying complementing (non-allelic) temperature-sensitive growth mutations ( $t s g$ ). Rare diploids formed between these strains were selected by growth at the non-permissive temperature $\left(27^{\circ} \mathrm{C}\right)$. While this procedure has been successfully and routinely adopted by several laboratories, it has two major disadvantages that make the finding of an alternative system desirable. First, any particular $t s g$ mutation is not generally distinguishable from any other. It is therefore difficult to ascertain by inspection whether haploid segregants from a diploid selected by the Tsg method possess one, or other, or both tsg mutations. Instead linkage or complementation analysis is necessary to identify the $t s g$ markers (Kessin, Williams \& Newell, 1974). This is tedious for routine work and not all tsg mutations are satisfactorily linked to known markers. Secondly, the possession of $t s g$ mutations by haploid strains makes it technically difficult to introduce developmental temperature-sensitive mutations. Because $t s g$ mutations are rare it is also not routinely feasible to select the $t s g$ mutation after the developmental temperature-sensitive mutation.

A possible alternative selection procedure, using drug-resistant mutations has been tried by several groups. Unfortunately all reports so far, except one (Fukui \& Takeuchi, 197I), indicate that resistance to a wide range of drugs tested is recessive (Sinha \& Ashworth, I969; Katz \& Sussman, 1972; Loomis, I97I ; Williams et al., 1974b; Wright, Williams \& Newell, unpublished). Diploids formed from pairs of haploids, each resistant to different drugs, are consequently sensitive to both drugs and while this system has been successfully used for selection of haploids from heterozygous diploids (Katz \& Sussman, 1972; Williams et al., $1974 b$ ), it cannot generally be used for the initial selection of the diploids. We therefore decided to look for mutants that were sensitive to drugs to which the wild type was resistant (rather than the converse) and to see if complementation of pairs of such mutants could be used to select diploids.

This paper shows that drug-sensitive mutants can, indeed, be used for diploid selection. 
In addition we report an extension of this principle to selection using sensitivity of slime mould growth to the bacterium Bacillus subtilis. This 'bsg' system has proved valuable for routine diploid isolation and can be used in conjunction with tsg mutations to avoid the two difficulties of double $t \mathrm{sg}$ selection mentioned above.

\section{METHODS}

Strains. All strains of Dictyostelium discoideum are derived from the NC4 isolate of Raper (I935). Strain NP20 is a spontaneous mutant resistant to $500 \mu \mathrm{g}$ cycloheximide $\mathrm{ml}^{-1}$ (cycAI). Strain AX3 is a mutant capable of growth axenically (Loomis, 197I). Strain NPI94 is a Bacillus subtilis-sensitive derivative of NP20 (cycAI, bsg). Strain X22 is a growth temperaturesensitive strain $(t s g D, t s g E)$ capable of growth on $2 \%(\mathrm{v} / \mathrm{v})$ methanol $(a c r A)$ and produces white, round spores (whi, sprA) (Williams \& Newell, 1976). The Enterobacter (formerly Aerobacter) aerogenes strain has been described previously (Williams \& Newell, I976). Bacillus subtilis 36. I is a non-sporulating mutant showing a very low reversion rate, obtained from Professor J. Mandelstam and Dr M. Young (University of Oxford).

Isolation of drug-sensitive mutants. Drug concentrations that caused about a $10 \%$ reduction in the rate of expansion of clones of $\mathrm{Ax} 3$ on plates were used for mutant selection: these were $0.3 \%(\mathrm{v} / \mathrm{v})$ for methanol and $100 \mu \mathrm{g} \mathrm{ml}^{-1}$ for cycloheximide. Drug-sensitive mutants were found by screening amoebae mutagenized with $N$-methyl- $N^{\prime}$-nitro- $N$-nitrosoguanidine (NTG) to Io \% survival (Kessin et al., I974).

Isolation of mutants sensitive to Bacillus subtilis 36.I. Strain NP20 was mutagenized with NTG to $20 \%$ survival and, after dilution and plating with $E$. aerogenes to produce 30 clones per plate, about ${ }_{10}^{4}$ amoebae from each surviving clone were transferred manually using toothpicks on to replica plates inoculated with either E. aerogenes or B. subtilis 36. I. To prevent carry-over growth of $E$. aerogenes on the latter, $0.1 \mathrm{ml}$ dihydrostreptomycin sulphate solution ( $2 \mathrm{mg} \mathrm{ml}^{-1}$ ) was routinely added with the $B$. subtilis inoculum. Enterobacter aerogenes was completely inhibited by the final concentration of antibiotic but $B$. subtilis 36 . I was unaffected.

Fusion of strains bearing the bsg marker with strains bearing tsg markers. Amoebae of both strains to be fused were separately harvested from the edges of clones growing on lawns of E. aerogenes on nutrient SM agar plates (Mosses, Williams \& Newell, 1975) using a sterile platinum wire loop, and suspended in $5 \mathrm{ml}$ sterile salts solution (SS) (Sussman, 1966). The bacteria were removed by differential centrifugation at room temperature using three rapid spins of $1000 \mathrm{rev}$. $\mathrm{min}^{-1}$ for $2 \mathrm{~min}$. Amoebae were then resuspended in $\mathrm{SS}$ to $2 \times 10^{2} \mathrm{ml}^{-1}$. Portions $(0.5 \mathrm{ml})$ of these suspensions were added to wells of Linbro tissue culture dishes (Linbro FB-I6-24-TC; Biocult, Paisley, Renfrewshire) either as separate suspensions (controls) or mixed suspensions of strains (for fusion) together with $0.5 \mathrm{ml}$ per well of fusion mixture, to give a cell density of $\mathrm{I} \times 10^{7} \mathrm{ml}^{-1}$, and shaken at $\mathrm{I} 70 \mathrm{rev} . \mathrm{min}^{-1}$ in an orbital shaker at $22{ }^{\circ} \mathrm{C}$ for $17 \mathrm{~h}$. Fusion mixture contained: $60 \mathrm{~mm}-\mathrm{KH}_{2} \mathrm{PO}_{4} / \mathrm{K}_{2} \mathrm{HPO}_{4}$ buffer, $\mathrm{pH}$ 7.5 , $100 \mathrm{ml}$; dextran (66000 mol. wt), $15 \mathrm{~g}$; and dihydrostreptomycin sulphate, $200 \mathrm{mg}$. The solids were gently dissolved in the buffer and filter-sterilized. The dextran seemed to promote a consistent rate of fusion probably by its action in preventing big clump formation. After incubation, $0.1 \mathrm{ml}$ samples of the amoebal suspension were plated directly on to SM nutrient agar plates together with a $0.1 \mathrm{ml}$ inoculum of B. subtilis 36.I (prepared by suspending, in $5 \mathrm{ml} \mathrm{SS}$, five loopfuls of bacteria from an overnight culture grown on a nutrient plate at $37^{\circ} \mathrm{C}$ ) and $\mathrm{O} \cdot \mathrm{I} \mathrm{ml}$ of filter-sterilized dihydrostreptomycin sulphate $\left(2 \mathrm{mg} \mathrm{ml} \mathrm{m}^{-1}\right)$. The plates were incubated at $27 \pm 0.2{ }^{\circ} \mathrm{C}$ for 4 days or until the diploid clones were large 
enough to be subcultured on to E. aerogenes plates for further testing. Some strains of $D$. discoideum did not develop on B. subtilis plates, showing instead an aggregateless phenotype; thus $D$. discoideum should not be kept as stocks on these plates for longer than a few days. Fusion of haploid strains without $b s g$ by double $t s g$ complementation can also be very satisfactorily performed using the fusion mixture described above and B. subtilis plates incubated at $27^{\circ} \mathrm{C}$. In this way, conditions of fusion and diploid selection can be standardized for all $b s g / t s g$ and $t s g / t s g$ fusions.

\section{RESULTS}

Isolation of drug-sensitive mutants and their use for diploid isolation. It seemed wise at the outset to use drugs with known effectiveness on the slime moulds and at concentrations that were not greatly inhibitory for the wild type. For this reason, methanol and cycloheximide were chosen initially. Mutants of $\mathrm{Ax}_{3}$ that were sensitive to these drugs were found by screening clones of mutagenized amoebae. From about I0oo clones tested three methanolsensitive mutants and two cycloheximide-sensitive mutants were isolated. Inocula of $10^{4}$ amoebae of the mutants formed no visible growth in 6 days in the presence of the drugs, but formed 10 to $20 \mathrm{~mm}$ diam. clones in their absence. None of the mutants showed sensitivity to both methanol and cycloheximide.

The dominance or recessiveness of the mutants was determined by incubating pairs of methanol-sensitive and cycloheximide-sensitive strains together under conditions promoting cell fusion, using the method described previously (Williams et al., I974a), and then plating on to medium containing both methanol and cycloheximide at a density of $10^{6}$ amoebae per plate. Controls indicated that the parental strain $\mathrm{AX} 3$ plated with $100 \%$ efficiency on this double-drug medium but that none of the sensitive mutants grew under these conditions. Amoebae from mixed incubations of methanol-sensitive and cycloheximide-sensitive mutants yielded doubly drug-resistant clones on methanol plus cycloheximide plates in all four combinations attempted. The diploid character of the clones was confirmed by determination of the shape and size of the spores produced by the growing clones; the diploids possessed characteristically curved spores which were approximately twice the volume of the haploids. We conclude that the mutations to methanol and cycloheximide sensitivity are both recessive to their wild-type alleles.

Isolation of mutants sensitive to Bacillus subtilis. Although the methanol-sensitive and cycloheximide-sensitive mutants indicated that the principle of diploid selection using sensitive mutants was tenable, these drugs were not suitable for routine diploid selection (see Discussion). Thus a search was carried out for other types of sensitive mutants.

Sensitivity to Bacillus subtilis 36. I was investigated because it was noticed that this bacterium was used as a food source at a slightly lower rate than E. aerogenes, Escherichia coli B or Salmonella typhimurium LT2. After testing 8750 mutagenized clones for sensitivity to $B$. subtilis, six mutants were isolated that were able to grow on $E$. aerogenes but failed to grow on $B$. subtilis 36 . I. (Controls indicated that the sensitivity was not due to other components such as the dihydrostreptomycin added with the $B$. subtilis inoculum). One of these mutants (NP194) seemed highly suitable for genetic work because of the absence of any slow leaky growth on the selection plates and its low reversion frequency $\left(<2 \times 10^{-7}\right)$.

Selection of diploids with B. subtilis-sensitive mutant NPI94. The recessive nature of the mutation for $B$. subtilis sensitivity (phenotype, Bsg; locus, $b s g$ ) and its use for selection of heterozygous diploids were tested by an experiment involving fusion of strains NPI94 $(c y c A I, b s g)$ and $\mathrm{x} 22(t s g E, \operatorname{spr} A$; whi, acr $A, t s g D)$. After $\mathrm{I} 7 \mathrm{~h}$ fusion, using the improved fusion technique (see Methods), amoebae were spread on to $B$. subtilis plates and incubated 
Table I. Genotypes and frequencies of haploid segregants of diploid DP746

Diploid DP746 was formed by fusion between strain NPI94 carrying the $b s g$ marker and strain x22 carrying a tsg marker using the selective technique described in Methods.

Linkage group...

DP746 $\left\{\begin{array}{l}\text { NPI94 } \\ \text { X22 }\end{array}\right.$

Haploid segregants

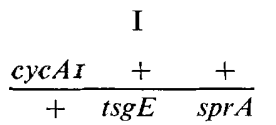

\begin{tabular}{ccc}
$c y c A I$ & + & + \\
\hline$c y c A I$ & + & + \\
\hline$c y c A I$ & + & + \\
\hline$c y c A I$ & + & + \\
\hline+ & $t s g E$ & $\operatorname{spr} A$ \\
\hline+ & $t s g E$ & $\operatorname{spr} A$ \\
\hline
\end{tabular}

II

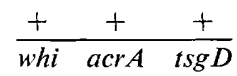

\begin{tabular}{ccc}
+ & + & + \\
\hline+ & + & + \\
\hline whi & acr $A$ & $t \operatorname{sg} D$ \\
\hline whi & acr $A$ & $t \operatorname{tsg} D$ \\
\hline whi & acr $A$ & $t s g D$ \\
\hline whi & acr $A$ & $t \operatorname{tsg} D$ \\
\hline
\end{tabular}

Unassigned

$\frac{b s g}{+}$

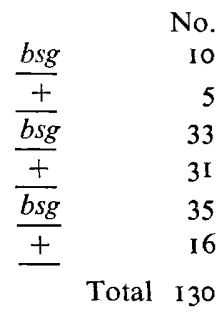

at $27{ }^{\circ} \mathrm{C}$ for 4 to 8 days. Controls using amoebae of either X22 or NPI94 incubated separately in fusion mixture did not form clones on any of 10 plates bearing $10^{6}$ amoebae per plate, even after 8 days. Amoebae from mixed incubation of X22 and NPI94, however, consistently showed about 20 clones per plate on each of 20 plates inoculated with $10^{6}$ amoebae per plate, indicating a frequency of diploid formation of $2 \times 10^{-5}$. Several such clones were isolated, recloned and their ploidy confirmed by analysis of spore shape and size. [The heterozygous diploid (spr $\mathrm{A} /+$ ) has a characteristically large oval shape.] Amoebae from one of these clones (DP746) were then plated at $5 \times 10^{3}$ amoebae per plate on to nutrient agar containing methanol $(2 \%, \mathrm{v} / \mathrm{v})$ or cycloheximide $\left(500 \mu \mathrm{g} \mathrm{ml}^{-1}\right)$ to resegregate haploids (Williams et al., 1974a). In the 130 segregant clones examined, sensitivity to B. subtilis segregated independently of resistance to cycloheximide or methanol/acriflavin (Table I).

We conclude that the $b s g$ marker is recessive, readily allows heterozygous diploid selection in amoebae in combination with $t s g$ markers and segregates independently of markers on linkage groups I and II.

\section{DISCUSSION}

The initial postulate that drug-sensitive mutations used in pairs might enable diploids to be selected, was borne out by the isolation of doubly resistant diploids on plates containing both methanol and cycloheximide. While this provided the necessary impetus to search for other types of sensitive mutant, it was not (nor was it intended to be) a routinely workable system. Most of the other laboratory stocks of $D$. discoideum used for genetic studies carry markers for resistance to cycloheximide or methanol/acriflavin. Furthermore the mutants isolated, particularly those for methanol sensitivity, were all slow-growing strains that showed a partially defective developmental ability. They were not, therefore, good parental stocks in which to introduce developmental mutations. In contrast, strains bearing the $b s g$ marker showed none of these undesirable qualities. They were generally indistinguishable from their parental strain in growth rate and they developed normally.

The use of haploids carrying the $b s g$ marker, together with $t s g$ bearing haploids, to select diploids, has the following advantages. First, the low reversion rate of Bsg strains gives a negligible background of revertants on $B$. subtilis plates. Diploids have been selected in over 50 separate fusion experiments using a variety of Tsg haploids as fusion partners. The $B$. subtilis lawn is very opaque and the growing clones produce neat transparent plaques in 
about 4 days, hence diploids can be selected earlier and more easily than with the $t \mathrm{sg} / \mathrm{tsg}$ fusions using $E$. aerogenes plates at $27^{\circ} \mathrm{C}$. Secondly, diploids synthesized in this way carry only one tsg mutation and there is therefore no ambiguity as to which tsg mutation is present in subsequently segregated haploids. Thirdly, Bsg strains are wild type with regard to their response to temperature, and temperature-sensitive mutations that are specific for development may therefore be readily selected. Genetic studies can then be performed by fusing the Bsg strains carrying developmental mutations to a well-marked master strain carrying a single $t s g$ marker. Such a master strain is currently being produced.

The bsg marker can also be used to perform other genetic manipulations that are otherwise difficult or impossible. For example, if a temperature-sensitive marker such as $\operatorname{tsg} E$ or $\operatorname{tsg} D$ is not required in a certain strain while adjoining markers (such as $\operatorname{spr} A$, whi, acr $A$ ) are needed, it is generally difficult to eliminate the $t s g$ marker on its own. Simple reversion of the $t s g$ marker would render the resultant strain temperature-resistant and consequently useless for subsequent diploid formation. If the $b s g$ marker is also present, however, the revertant strain isolated can subsequently be fused to other strains and the markers adjoining the reverted $t s g$ mutation used in other crosses. In addition, an interesting type of apparent revertant of $t s g D$ has been found that retains the $t s g$ marker but gains an unlinked suppressor mutation; such suppressors may be conveniently analysed with the aid of the $b s g$ fusion system.

This work was supported by grants from the Science Research Council to P.C.N. and from the U.S. National Institutes of Health, Division of General Medical Sciences to D.I.R. (fellowship no. 5-F32-GMo5328-02). We thank Dr M. Young and Professor J. Mandelstam for the gift of B. subtilis 36.1 .

\section{REFERENCES}

Brody, T. \& Williams, K. L. (1974). Cytological analysis of the parasexual cycle in Dictyostelium discoideum. Journal of General Microbiology 82, 37 [-383.

FukUi, Y. \& TAKEUCHI, I. (I97I). Drug resistant mutants and appearance of heterozygotes in the cellular slime mould Dictyostelium discoideum. Journal of General Microbiology 67, 307-3 7 .

Katz, E. R. \& Sussman, M. (1972). Parasexual recombination in Dictyostelium discoideum: selection of stable diploid heterozygotes and stable haploid segregants. Proceedings of the National Academy of Sciences of the United States of America 69, 495-498.

Kessin, R. H., Williams, K. L. \& Newell, P. C. (1974). Linkage analysis in Dictyostelium discoideum using temperature-sensitive growth mutants selected with bromodeoxyuridine. Journal of Bacteriology rig, $776-783$.

Loomis, W. F. (1969). Temperature sensitive mutants of Dictyostelium discoideum. Journal of Bacteriology 99 , $65-69$.

Loomis, W. F. (I97I). Sensitivity of Dictyostelium discoideum to nucleic acid analogues. Experimental Cell Research 64, 484-486.

Mosses, D., Williams, K. L. \& Newell, P. C. (1975). The use of mitotic crossing-over for genetic analysis in Dictyostelium discoideum: mapping of linkage group II. Journal of General Microbiology 9o, $247-259$.

Pontecorvo, G. \& Käfer, E. (1958). Genetic analysis based on mitotic recombination. Advances in Genetics 9, 7I-104.

RAPER, K. B. (I935). Dictyostelium discoideum, a new species of slime mold from decaying forest leaves. Journal of Agricultural Research 50, $135-147$.

Sinha, U. \& ASHWORTH, J. M. (I969). Evidence for the existence of elements of a parasexual cycle in the cellular slime mould Dictyostelium discoideum. Proceedings of the Royal Society Br73, 53 I-540.

Sussman, M. (1966). Biochemical and genetic methods in the study of cellular slime mould development. In Methods in Cell Physiology, vol. 2, pp. 397-410. Edited by D. Prescott. New York: Academic Press.

Williams, K. L. \& Newell, P. C. (I976). A genetic study of aggregation in the cellular slime mould Dictyostelium discoideum using complementation analysis. Genetics $\mathbf{8 2}, 287-307$.

Williams, K. L., Kessin, R. H. \& Newell, P. C. (1974a). Genetics of growth in axenic medium of the cellular slime mould Dictyostelium discoideum. Nature, London 247, 142-143.

Williams, K. L., Kessin, R. H. \& Newell, P. C. (1974b). Parasexual genetics in Dictyostelium discoideum: mitotic analysis of acriflavin resistance and growth in axenic medium. Journal of General Microbiology 84, 59-69. 